\title{
ARTICLE
}

\section{Radioactivity evaluation of the secondary sodium in DRACS of the Japan Sodium-cooled Fast Reactor}

\author{
Kenji Sasaki $^{\mathrm{a}^{*}}$, Katsuaki Naito ${ }^{\mathrm{b}}$, Shigeo Ohki ${ }^{\mathrm{c}}$, Tsutomu Okubo ${ }^{\mathrm{c}}$ and Shoji Kotake ${ }^{\mathrm{d}}$ \\ ${ }^{a}$ Mitsubishi FBR Systems, Inc., 2-34-17, Jingumae, Shibuya-ku, Tokyo, 150-0001, Japan; ${ }^{b}$ Ctec, Inc., 2-34-17, Jingumae, Shibuya-ku, \\ Tokyo, 150-0001, Japan; ' Japan Atomic Energy Agency, 4002, Narita-cho, O-arai-machi, Ibaraki, 311-1393, Japan; ${ }^{d}$ The Japan \\ Atomic Power Company, 1-1, Kanda-Mitoshiro-cho, Chiyoda-ku, Tokyo, 101-0053, Japan
}

This paper evaluates the secondary sodium activation in Direct Heat Exchanger (DHX), the radioactivity density and the dose rate around the secondary sodium pipes in Direct Reactor Auxiliary Cooling System (DRACS) of the Japan Sodium-cooled Fast Reactor (JSFR), by improving the exactness of a shielding calculation model with the Monte Carlo methodology. From this result, the prospects to meet the shielding design requirements for radioactivity free areas are obtained.

Keywords: JSFR; DRACS; DHX; secondary sodium; shielding design; radioactivity free area; Na-24 radioactivity; shielding analysis; MCNP; JENDL-3.3

\section{Introduction}

The Fast Reactor Cycle Technology Development (FaCT) project has been pursued the commercialization of a fast reactor cycle system around 2050 under the cooperation of MEXT (Ministry of Education, Culture, Sports, Science and Technology), METI (Ministry of Economy, Trade and Industry), utilities, vendors and JAEA (Japan Atomic Energy Agency). As results of the FaCT phase I which has finalized in 2010, the key technologies for the Japan Sodium-cooled Fast Reactor (JSFR) have been evaluated whether those are feasible to be installed in the demonstration JSFR which is planned to be operated from 2025[1-3].

Major specifications and the bird's-eye view of the commercial JSFR are shown in Figure 1 and Table 1, respectively. JSFR aims to be able to compete economically by compact design and achieves the Generation IV reactor goals by adapting the following key technologies:

(1) High burn-up fuel with the Oxide Dispersion Strengthened (ODS) cladding material

(2) Safety enhancement with a self-actuated shutdown system and a re-criticality free core

(3) Compact reactor system (Reactor Vessel: RV) adopting a hot vessel and in-vessel fuel handling with a Upper Inner Structure with a slit (slit UIS) and an advanced Fuel Handling Machine (FHM)

(4) Two-loop cooling system with a large diameter piping made of the Mod. 9Cr-1Mo steel

(5) Integrated component of a Pump and an Intermediate

*Corresponding author. Email: k_sasaki@mfbr.mhi.co.jp
Heat Exchanger (Pump-IHX)

(6) More highly reliable Steam Generator (SG) with double-walled tubes

(7) Natural circulation by the Decay Heat Removal Systems (DHRS)

(8) Simplified fuel handling system

(9) Containment vessel made of steel plate reinforced concrete

(10) Advanced seismic isolation system

The Direct Reactor Auxiliary Cooling System (DRACS), which is one of the systems configuring DHRS, is one of the most important safety systems of JSFR for cooling the core in case of serious accidents.

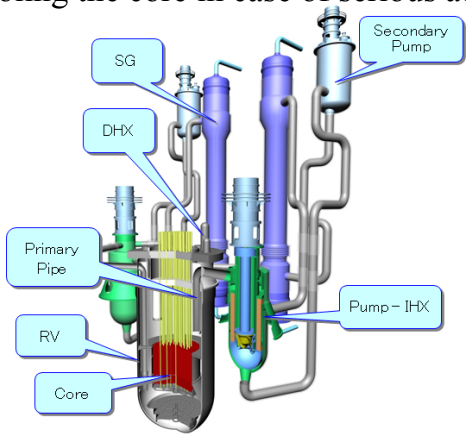

Figure 1. Bird's-eye view of JSFR.

Table 1. JSFR major specifications.

\begin{tabular}{|c|c|}
\hline Items & Value \\
\hline Electric Output & $1500 \mathrm{MWe}$ \\
\hline Thermal Output & $3530 \mathrm{MWth}$ \\
\hline Fuel Type & MOX \\
\hline Configuration & Loop \\
\hline Number of Loop & 2 \\
\hline Primary Sodium Temperature & 550 deg-C \\
\hline Reactor Vessel Material & 316 Stainless Steel \\
\hline Piping Material & Mod. 9Cr-1Mo Steel \\
\hline
\end{tabular}


This study is focusing on the secondary sodium activation in DHX for confirming the feasibility of the DRACS shielding design.

The conceptual view of DRACS is shown in Figure 2. DRACS consists of DHX, the secondary sodium pipes, the air cooler, the expansion tank, etc. DHX is required to be located near the core, but the secondary sodium pipes, the expansion tank and the air cooler are required to be located in radioactivity free areas away from the core for facilitating the repair and maintenance.

In addition, the core fuel assembly with inner duct structure (FAIDUS) shown in Figure 3 is adopted as a measure for early discharge of the molten fuel. For shielding design, it is not preferred because it acts to increase the secondary sodium activation in DHX by neutron streaming through it. There are the following reactions of the secondary sodium activation: Na-23 (n, r) Na-24, Na-23 (n,2n) Na-22, and Na-23 (n, p) Ne-23. The major reaction is Na-23 $(\mathrm{n}, \gamma) \mathrm{Na}-24$.

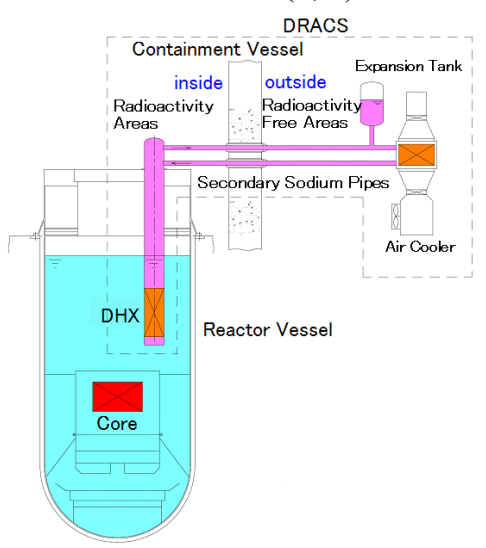

Figure 2. Conceptual view of DRACS.

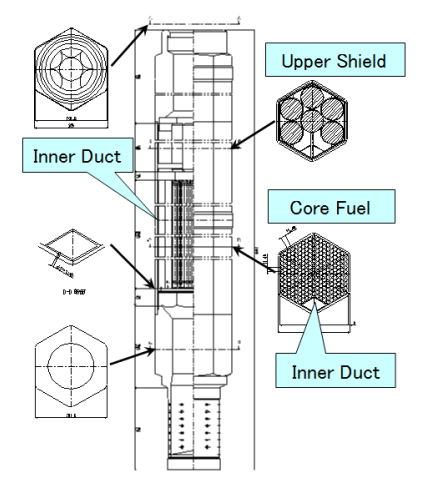

Figure 3. Conceptual view of FAIDUS.

Although two-dimensional discrete ordinate transport code, DORT [4], has been used as the current fast reactor shielding design method, this code was unsuitable to model accurately the complex configurations and to decrease the correction factors for modeling. From these reasons, a Monte Carlo calculation code, MCNP [5], was nominated as the new calculation method for resolving these problems.

However MCNP has the following problems as compared with DORT in order to calculate the radiation flux from a large-scale model with deep penetration:

(1) Central Processing Unit (CPU) time is long.
(2) Input data is complex.

(3) Visualization of calculation results is complex.

Comparison between MCNP and DORT methods is shown in Table 2.

In this study, it was tested whether MCNP calculations obtained the reliable solutions, in other words the Fractional Standard Deviations (FSDs) were small enough. Namely, the MCNP calculations focusing the followings were performed:

- whether the FSD of the flux around the core was below $10 \%$ by the surface crossing method,

- whether the FSD of the secondary sodium activation in DHX was below $10 \%$ by the track length method,

- whether the FSD of the dose rate around the secondary sodium pipes in DRACS was below $5 \%$ by the point detector method.

Table 2. Comparison between MCNP and DORT methods.

\begin{tabular}{|c|c|c|}
\hline Items & \multicolumn{2}{|c|}{ Methods } \\
\hline Used Code & MCNP & DORT \\
\hline Modeling of Complex Configurations & Possible & Impossible \\
\hline Correction Factors for Modeling & Small & Large \\
\hline CPU Time & Long & Short \\
\hline Input Data & Complex & Simple \\
\hline Visualization of Calculation Result & Complex & Simple \\
\hline
\end{tabular}

\section{Shielding design requirements for DRACS}

The shielding design requirements of $\mathrm{Na}-24$ density and dose rate in radioactivity free areas are based on the appended table 1 in the notification No. 74 of MEXT and the notification No. 295 of METI. The limited values of $\mathrm{Na}-24$ density and dose rate are the followings:

(1) Na-24 density : below $10 \mathrm{~Bq} / \mathrm{g}$

(2) Dose rate : below $2.6 \mu \mathrm{Sv} / \mathrm{h}$

\section{Calculation model and methods}

\subsection{Cross sections for MCNP}

The cross sections for MCNP calculation were produced by using the NJOY [6] code from the evaluated nuclear data library, JENDL-3.3 [7]. Thermal neutron scattering law $S(\alpha, \beta)$ was considered for the $\mathrm{Zr}-\mathrm{H}$ shields around the core.

\subsection{Evaluation around core and $\mathrm{DHX}$}

The MCNP calculation model for evaluating the secondary sodium activation in DHX by neutrons leaked from the core represents the complex configurations of the core, core barrel, DHX, RV, guard vessel $(\mathrm{GV})$ and reactor cavity wall which covers radially $1552 \mathrm{~cm}$ length from wall to wall, and axially $3050 \mathrm{~cm}$ length from the bottom to the top (the reactor head access area). The end of the equilibrium cycle where all control rods are withdrawn is chosen as the core model because the neutron leakage flux from the core is the highest in the cycle. This model is shown in Figure 4.

In order to satisfy the shielding design requirements for DRACS, the following radiation shielding measures 
are performed:

(1) The radiation shielding materials are set outside DHX as shown in Figure 5. The radiation shielding materials are natural $\mathrm{B}_{4} \mathrm{C}$ and the thicknesses are from $12 \mathrm{~cm}$ to $38 \mathrm{~cm}$.

(2) DHX is set as far as possible from the core for decreasing the neutron flux field.

(3) As the shielding measures for preventing neutron streaming through the inlet and outlet opening areas of the first sodium flow, the entrance position of the primary sodium is set up and the lower side radiation shield is extended as much as possible.

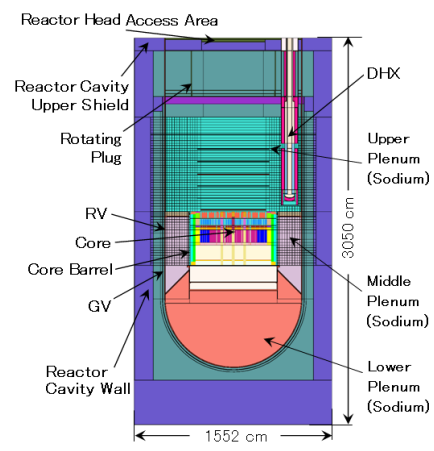

(a) Vertical sectional view

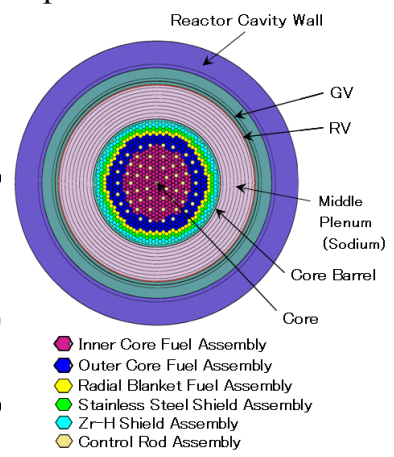

(b) Horizontal sectional view
Figure 4. Calculation model around core and DHX.

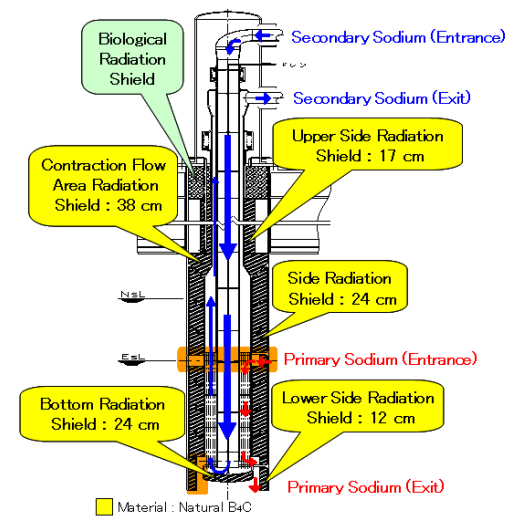

Figure 5. Radiation shielding measure of DHX.

The calculations were carried out in order to estimate the FAIDUS effect on neutron streaming through the inner duct and the $S(\alpha, \beta)$ effect on thermal neutron scattering in $\mathrm{Zr}-\mathrm{H}$ shields. Namely, the following two MCNP hexagonal core geometry models which treated in detail the reactor components and the DHX structure shown in Figures 4 and 5 were created and calculated as the fixed source problem with the cell importance technique for variance reduction:

(1) MCNP heterogeneous model which separated the inner duct in the core fuel assembly but homogenized in other assemblies.

(2) MCNP homogeneous model which mixed materials in the core fuel assembly in the same way as other assemblies.

MCNP heterogeneous model were gathered several inner ducts in one place for estimating conservatively neutron streaming as shown in Figure 6.

Three MCNP calculations have been performed in order to consider the FAIDUS and $\mathrm{S}(\alpha, \beta)$ effects by the surface crossing method and have been compared with the DORT calculation.

Further, the calculation of the MCNP heterogeneous model that considered both the FAIDUS and $S(\alpha, \beta)$ effects has been performed by the track length method for evaluating the secondary sodium activation in DHX. The Na-24 density in DRACS has been calculated from the Na-24 inventory in DHX which was estimated on the basis of this calculation.

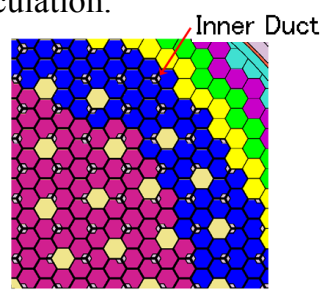

Figure 6. Extended figure of core center plane.

\subsection{Evaluation around secondary sodium pipe}

The MCNP calculation model of the dose rate around the secondary sodium pipe is shown in Figure 7. This model represents simple configurations of the secondary sodium, secondary sodium pipe, nitrogen gas, enclosure pipe and heat insulating material. It covers $80.04 \mathrm{~cm}$ in the diameter and axially a $1000 \mathrm{~cm}$ length. This calculation has used the Na-24 density as the radioactive source and has been carried out by the point detector method.

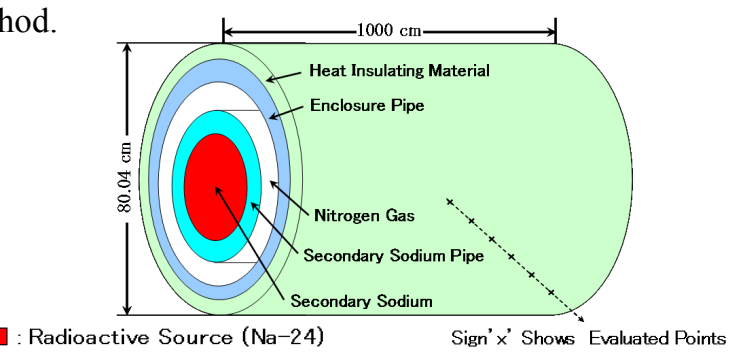

Figure 7. Calculation model of dose rate around secondary sodium pipe.

\section{Calculation results}

The calculation results are shown in Table 3. The $\mathrm{Na}-24$ inventory in DHX is $1.64 \times 10^{8} \mathrm{~Bq}$ and the FSD is $6.7 \%$. The former includes the uncertainty of $3 \sigma$ (FSD). The MCNP calculation to evaluate the Na-24 inventory needed 11 billion histories, which took 120 days (CPU Time) with 5 CPUs (EWS-IBM).

The following correction factors are added to the calculated value $(6.0 \mathrm{~Bq} / \mathrm{g})$ of the $\mathrm{Na}-24$ density in DRACS:

(1) Uncertainty of generated neutrons from the blanket fuel region was set at $20 \%$ with reference to the preceding reactor shielding evaluation. The correction factor (1.2) shown in Table 3 was set for obtaining conservative results.

(2) The MCNP calculations on the experiments of Oak Ridge National Laboratory (ORNL) [8] [9] were performed with parameterizing both the bonner ball 
detectors and the thickness of the sodium and $\mathrm{B}_{4} \mathrm{C}$ shields. From these results, the ratios of experiment (E) and calculation (C) were evaluated as 0.88 [10] and 1.15 for the sodium and $\mathrm{B}_{4} \mathrm{C}$ shields, respectively. As a result, the corrected $\mathrm{Na}-24$ density in DRACS has been obtained as $7.3 \mathrm{~Bq} / \mathrm{g}$.

The MCNP calculation value for the dose rate around the secondary sodium pipe is $0.8 \mu \mathrm{Sv} / \mathrm{h}$ and the FSD is $1.3 \%$. The MCNP calculation to evaluate the dose rate needed 10 million histories, which took only 14 minutes (CPU Time) with 1 CPU (EWS-IBM).

Table 3. Calculation results.

\begin{tabular}{|c|c|c|c|c|}
\hline \multicolumn{3}{|c|}{$\mathrm{Na}-24$ Inventory in DHX (Bq) } & $1.64 \times 10^{8}$ & $(6.7 \%)^{* 1}$ \\
\hline \multicolumn{3}{|c|}{ Na-24 Density in DRACS $(\mathrm{Bq} / \mathrm{g})$} & \multicolumn{2}{|c|}{6.0} \\
\hline \multirow{3}{*}{$\begin{array}{c}\text { Correction } \\
\text { Factor }\end{array}$} & \multicolumn{2}{|c|}{ Generated Neutron from Core } & \multicolumn{2}{|c|}{1.2} \\
\hline & \multirow{2}{*}{$\begin{array}{c}\text { Expriment } \\
\text { /Calculation }{ }^{* 2}\end{array}$} & Sodium & \multicolumn{2}{|c|}{0.88} \\
\hline & & $\mathrm{B}_{4} \mathrm{C}$ Shield in DHX & \multicolumn{2}{|c|}{1.15} \\
\hline \multicolumn{3}{|c|}{ Corrected Na-24 Density $(\mathrm{Bq} / \mathrm{g})$} & \multicolumn{2}{|c|}{7.3} \\
\hline \multicolumn{3}{|c|}{ Dose Rates $(\mu \mathrm{Sv} / \mathrm{h})$} & 0.8 & $(1.3 \%)^{*}$ \\
\hline
\end{tabular}

\section{Discussion}

Figure 8 shows the relationship between the position of DHX and the total neutron flux contour which is calculated around the core by the DORT code. The DORT calculation has been carried out with 100 neutron energy groups, a P3 scattering expansion and a S117 asymmetric angular quadrature set in R-Z (cylindrical) geometry, where the $S(\alpha, \beta)$ effect was not considered. From this figure, DHX is found to be located in the very high neutron flux field from $10^{8}$ to $10^{4} \mathrm{n} / \mathrm{cm}^{2} \mathrm{~s}$.

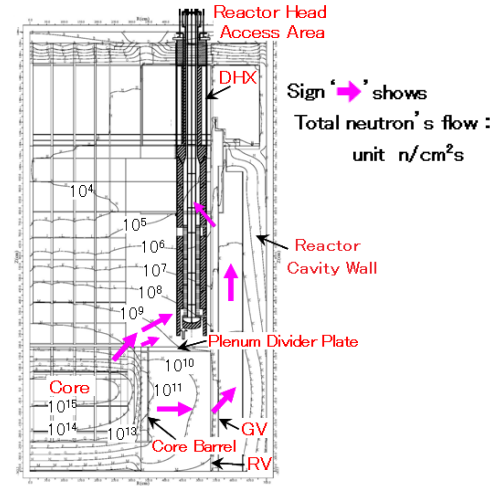

Figure 8. Relationship between position of DHX and total neutron flux by DORT calculation.

Figure 9 shows the comparison of total neutron flux distribution by the DORT and MCNP calculations.

The upper in Figure 9 shows the FAIDUS effect at the top of core assembly. The total neutron flux of the heterogeneous model increases 5.7 times from the homogeneous models.

The lower in Figure 9 shows the core (hexagonal /cylindrical) geometry effect and the $S(\alpha, \beta)$ effect at the core center. It is found that the modeling effect between the cylindrical core and the hexagonal core is significant from the comparison of the DORT and the MCNP calculations. Furthermore the followings are found:
(1) The $S(\alpha, \beta)$ effect at the core barrel is large from the results of the total, epi-thermal $(0.414-1.125 \mathrm{eV})$ and thermal neutron fluxes. The MCNP model results considered the $S(\alpha, \beta)$ effect are 1.5, 1.6 and 2.1 times higher, respectively, than those of MCNP models not considered the $S(\alpha, \beta)$ effect.

(2) Otherwise the $S(\alpha, \beta)$ effect at the RV, GV and liner before the reactor cavity wall is small from the results that each region's fluxes of both MCNP models are almost the same.

From these results, the $\mathrm{S}(\alpha, \beta)$ effect in the $\mathrm{Zr}-\mathrm{H}$ shields is not affect the evaluation of the secondary sodium activation in DHX.

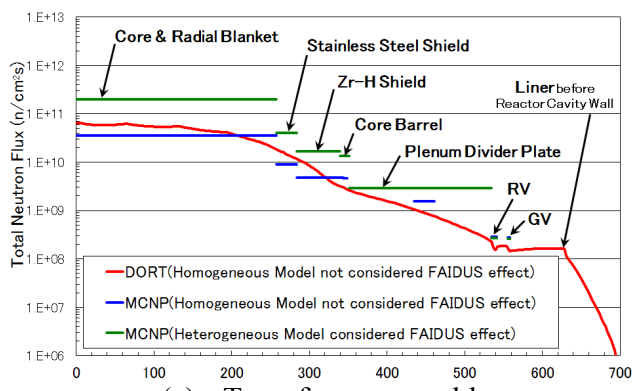

(a) Top of core assembly

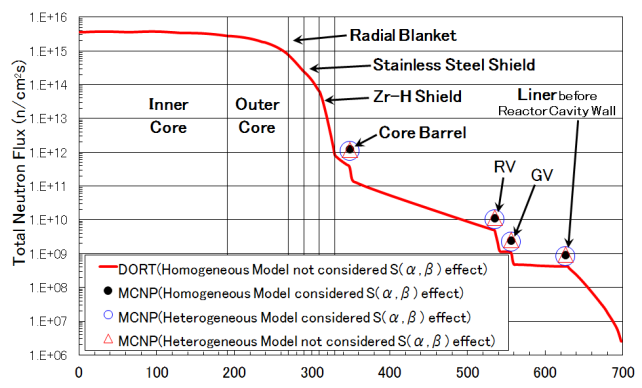

(b) Core center

Figure 9. Comparison of total neutron flux distribution between DORT and MCNP.

\section{Conclusion}

The conclusions of this study are as follows:

(1) A Monte Carlo calculation code, MCNP, is used in the shielding design of JSFR. The evaluated values (FSD) of the $\mathrm{Na}-24$ density and dose rate are $7.3 \mathrm{~Bq} / \mathrm{g}$ $(6.7 \%)$ and $0.8 \mu \mathrm{Sv} / \mathrm{h}(1.3 \%)$, respectively. The prospects to meet the shielding design requirements for radioactivity free areas are obtained.

(2) The total neutron flux at the top of core assembly increases 5.7 times by the FAIDUS effect.

(3) Since the modeling effect between cylindrical and hexagonal cores is significant at the core center, it is necessary to pay attention to the modeling of the core.

(4) Since the $S(\alpha, \beta)$ effect is small except at the core barrel, it does not affect the evaluation of the secondary sodium activation in DHX.

(5) In this study, it is confirmed that the MCNP calculation can model accurately the complex configurations for decreasing the correction factors and then can obtain the reliable solutions in which the FSD is small enough. 


\section{Future problem for MCNP calculation}

The verification and validation of this Monte Carlo methodology will be performed for the future shielding measurements plan in the prototype FBR Monju [11]. These results will be useful to improve the shielding analysis methods for future FBRs.

The problems for MCNP calculation to be solved in the shielding design of the commercial plant (this study: 1500 MWe JSFR) and the demonstration plant (recent study: 750 MWe JSFR) [12] are as follows:

(1) Reduction of calculation time is very important for using in a variety of shielding design. Applications of the software such as the weight window generator and density reduction method, and the strengthening of hardware along with speed up of computer machine are useful to achieve this purpose. The comparison of calculation efficiency between this study and recent study for evaluating the secondary sodium activation in DHX is shown in Figure 10. The 750 MWe JSFR is a scaled-down version of the 1500 MWe JSFR. Though the 750 MWe JSFR core is about half size of the 1500 MWe JSFR core, the both cores have the same systems and the same structures of the core component assemblies. The upper shields of core and blanket assemblies, stainless steel and $\mathrm{Zr}-\mathrm{H}$ shields of the 750 MWe JSFR have been modeled more in detail than those of the 1500 MWe JSFR. In the MCNP calculation for the 750 MWe JSFR, the EWS capacity has increased from 5 CPUs to $22 \mathrm{CPUs}$, then as a result the CPU time has reduced from 120 days to 17 days, and the history number has reduced from 11 billion to 1.5 billion, in order to obtain almost the same FSD value as before. As the ratio of the history number and the multiplication of the number of $\mathrm{CPU}$ and the CPU time has reduced from $4.6 \times 10^{7}$ to $4.0 \times 10^{6}$, it is concluded that the calculation efficiency has considerably improved.

(2) Simplification of input data is important in order to eliminate the error in calculation. The computer-aided design (CAD) / MCNP conversion interface code, GEOMIT [13], will be used for this purpose.

(3) Visualization of calculation results is important in order to be judged the validity timely and accurately. It is desirable to develop the contour of radiation flux distribution by using mesh based tally.

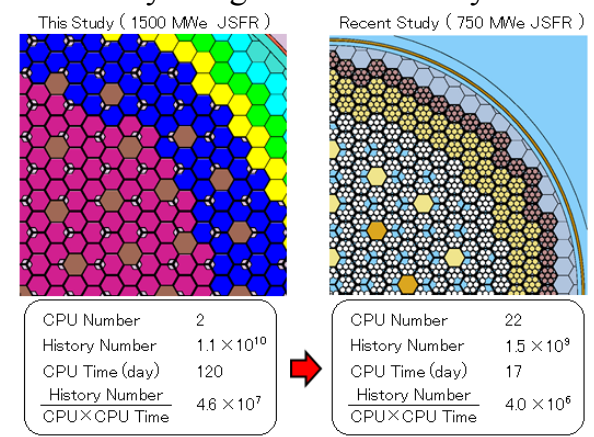

(Extended figures show the upper shield plane)

Figure 10. Comparison of calculation efficiency between this study and recent study.

\section{Acknowledgements}

This paper includes the outcome of collaborative study between JAEA and JAPC (that is the representative of nine electric utilities, Electric Power Development Co., Ltd. and JAPC) in accordance with the agreement on the development of a commercialized fast breeder reactor cycle system.

\section{References}

[1] Y. Chikazawa, H. Hayafune and Y. Aoto, Conceptual design for a large-scale Japan Sodium-cooled Fast Reactor (1) feasibility of key technologies, Proc. ICAPP 2011 (2011), Paper No. 11278.

[2] H. Yamano, S. Kubo and Y. Shimakawa, Conceptual design for a large-scale Japan Sodium-cooled Fast Reactor (2) safety design and evaluation in JSFR, Proc. ICAPP 2011 (2011), Paper No. 11219.

[3] T. Okubo, S. Ohki and M. Ogura, Conceptual design for a large-scale Japan Sodium-cooled Fast Reactor (3) core design in JSFR, Proc. ICAPP 2011 (2011), Paper No. 11345.

[4] TORT-DORT-PC two-and-three-dimensional discrete ordinate transport version 2.7.3, R.S.I.C, CCC-543, (1996).

[5] X-5 Monte Carlo Team, MCNP-A general Monte Carlo N-particle transport code version 5 volume I: overview and theory, LA-UR-03-198, (2005).

[6] NJOY99.0 code system for producing pointwise and multigroup neutron and photon cross sections from ENDF/B data, R.S.I.C, PSR-480, (2000).

[7] Japanese evaluated nuclear data library version 3 revision-3: JENDL-3.3, J. Nucl. Sci. Technol. 39, 1125, (2002).

[8] Final report on a benchmark experiment for neutron transport in thick sodium, ORNL-4880, (1974).

[9] Measurement for the LMR alternate shielding material experiment, ORNL-TM-9977, (1986).

[10] K. Sasaki, K. Naito and S. Ohki, Shielding design of JSFR evaluation of the ORNL sodium transmission measurements by using Monte Carlo code MCNP, Proc. 2011 Spring Meeting of J. At. Energy Soc. (2011), G15, p. 329. [in Japanese]

[11] K. Sasaki, S. Usami and T. Deshimaru, Evaluation of neutron streaming and future shielding measurement plan in the prototype FBR Monju, Proc. ICRS-9 (2000), Sup.1, pp.104-109.

[12] S. Maruyama, K. Kawasaki and S. Ohki, Study on FBR core concepts for the LWR-to-FBR transition period, Proc. GLOBAL 2009 (2009), Paper No. 9316.

[13] H. Nasif, F. Masuda and H. Morota, Development of CAD-MCNP interface program GEOMIT and its applicability for ITER neutronics design calculations, Nucl. Technol. 180, NT 10-10-119, (2012). 